\title{
Genetic variability of oil palm parental genotypes and performance of its' progenies as revealed by molecular markers and quantitative traits.
}

\begin{abstract}
Studies were conducted to assess the genetic relationships between the parental palms (dura and pisifera) and performance of their progenies based on nine microsatellite markers and 29 quantitative traits. Correlation analyses between genetic distances and hybrids performance were estimated. The coefficients of correlation values of genetic distances with hybrid performance were non-significant, except for mean nut weight and leaf number. However, the correlation coefficient of genetic distances with these characters was low to be used as predicted value. These results indicated that genetic distances based on the microsatellite markers may not be useful for predicting hybrid performance. The genetic distance analysis using UPGMA clustering system generated 5 genetic clusters with coefficient of 1.26 based on quantitative traits of progenies. The genotypes, DP16, DP14, DP4, DP13, DP12, DP15, DP8, DP1 and DP2 belonging to distant clusters and greater genetic distances could be selected for further breeding programs.
\end{abstract}

Keyword: Oil palm; Microsatellite markers; Quantitative characters; Correlation. 